\title{
Ischemia-modified albumin levels in children with asthma: a pilot study
}

\author{
Mahmut Dogru, M.D. ${ }^{a}$, Handan Akoglu, M.D. ${ }^{a}$, Muhammet F. Kilinckaya, M.D. ${ }^{b}$ and \\ Gozde Ulfer, M.D.c
}

\begin{abstract}
Introduction: Hypoxia may occur in the severe exacerbations of asthma. Ischemia-modified albumin (IMA) may increase in ischemia, in addition to oxidative stress and inflammation. The aim was to evaluate IMA levels in children during the asthma exacerbation and the asymptomatic period.

Populations and methods: Children with asthma who were followed up in our clinic were included and healthy children were selected as the control group. The severity of exacerbation was evaluated with Global Initiative for Asthma and Modified Pulmonary Index Score. Serum IMA levels were measured at the time of exacerbation and 4 weeks after treatment during asymptomatic period. Skin prick test and $C$ reactive protein (CRP) levels were measured.
\end{abstract}

Results: A total of 26 patients and 26 controls were included. Mean IMA level was $0.45 \pm 0.12$ absorbance units -ABSU- during asthma exacerbation and $0.32 \pm 0.08$ ABSU in the control group ( $\mathrm{p}=0.001)$. Mean IMA levels $(0.41 \pm 0.14$ ABSU) during the stable period were higher than the control group $(p=0.005)$. There was no difference in terms of IMA levels when patients were grouped according to anti-inflammatory treatment, upper respiratory tract infection before exacerbation, CRP levels or sensitivity of skin prick tests. However, IMA levels werehigher in patients with severe asthma exacerbation $(\mathrm{p}=0.009)$ in comparison with mild/moderate exacerbation. Positive correlation was observed between IMA levels and severity of exacerbation (r: 0.498, $\mathrm{p}=0.010$ ).

Conclusions: Asthmatic children had higher IMA levels than the control group, both in stable and exacerbated asthma. There was a positive relationship between IMA levels and severity of asthma exacerbation.

Key words: asthma, child, asthma exacerbation, hypoxia, ischemia-modified albumin.

http: / / dx.doi.org/10.5546/ aap.2018.eng.e522

To cite: Dogru M, Akoglu H, Kilinckaya M, Ulfer G. Ischemia-modified albumin levels in children with asthma: a pilot study. Arch Argent Pediatr 2018;116(4):e522-e528.

\section{INTRODUCTION}

Asthma is defined by the history of having respiratory symptoms such as wheeze, shortness of breath, chest tightness and cough that vary over time and in intensity, together with variable expiratory airflow limitation. Exacerbation of asthma is characterized by progressive decrease in lung function and progressive increase in symptoms. The main characteristic of an asthma exacerbation is airflow obstruction in the respiratory tract. Hypoxia caused by airway obstruction, can be evaluated by oxygen saturation measurement to assess the severity of asthma exacerbation. The aim of treatment is to rapidly relieve airflow obstruction and hypoxemia, address the underlying inflammatory pathophysiology, and prevent relapses. In the first step of treatment of asthma exacerbation in the emergency department, oxygen is administered by face mask to achieve and maintain percutaneous oxygen saturation between $94-98 \%{ }^{1}$

The terminal end of amino acid in albumin has the capacity to bind heavy metals (copper, nickel and cobalt). In pathological conditions such as hypoxia or acidosis a free radical damage that occurs in the case of ischemia decrease the binding capacity of metals to the N-terminal domain of albumin. Bar-Or et al. ${ }^{2}$ detected the decreased binding capacity of cobalt into the N-terminal domain of albumin in patients with myocardial infarction for the first time, and they developed the albumin cobalt binding test knows as "ischemia-modified albumin (IMA)". IMA has been extensively studied in ischemic heart diseases and the 
measurement has been approved by the Food and Drug Administration (FDA) to assess the extent of cardiac ischemia. Ischemia-modified albumin is accepted as a marker of oxidative stress. ${ }^{2-4}$ Besides diseases related with ischemia, IMA levels were found higher in patients with acute rheumatic fever, ${ }^{5}$ chronic obstructive pulmonary disease (COPD), ${ }^{6}$ inflammatory bowel disease ${ }^{7}$ and Behçet's disease ${ }^{8}$ compared $^{\prime}$ to controls. Asthma may cause hypoxia during an exacerbation but we have limited information about IMA levels in asthma. In Turkey, Karagol et al. ${ }^{9}$ evaluated IMA levels in asthma exacerbations and they found that IMA levels were higher in patients compared to controls. They also found no significant difference between IMA levels of controls and asthmatic patients which were evaluated 2 weeks after asthma exacerbations.

The aim of this study was to evaluate IMA levels of children in asymptomatic period and during asthma exacerbation in comparison with the IMA levels of healthy children.

\section{POPULATIONS AND METHODS}

Study population: asthmatic children consecutively admitted to the emergency department due to an asthma exacerbation between December 2015 and March 2016, were included. Patients with obesity (body mass index $>95 \mathrm{P})^{10}$ and patients with any chronic diseases such as cystic fibrosis or bronchiectasis were excluded from this study. Children without any allergic or chronic disease who were referred to the general pediatric outpatient clinic for routine screening and receiving blood samples for other tests (complete blood count, urea, creatinine, etc.) were taken as control group.

Study design: in this prospective, crosssectional study, patients were asked to fill a questionnaire during the first assessment. This questionnaire includes the demographic characteristics of patients, usage of antiinflammatory medicines, the presence of upper respiratory tract infection (URTI) symptoms before exacerbation, the number of exacerbations in the last year and the number of hospitalizations, and history of atopy.

First evaluation: patients were evaluated both clinically (Global Initiative for Asthma (GINA) and Modified Pulmonary Index Score (MPIS)) and with laboratory tests (IMA and C-reactive protein [CRP]) during asthma exacerbation. $5 \mathrm{ml}$ of serum of patients' blood were collected at the time of exacerbation and stored $-20^{\circ} \mathrm{C}$. $\mathrm{C}$-reactive protein (CRP) measurement was performed within 2 hours of collection at the time of exacerbation.

Second evaluation: the blood samples of patients for IMA were taken at least 4 weeks after the treatment of acute exacerbations and in the period when they were asymptomatic.

Ethical aspects: the ethical approval of Zeynep Kamil Woman and Children's Diseases Training and Research Hospital was taken: the aim and procedures of the study were explained to children and their families and their oral approval was taken. The study adhered to the principles of Helsinki Declaration.

\section{Clinical evaluation}

The severity of asthma exacerbation of patients was categorized by Global Initiative for Asthma (GINA) as mild/moderate and severe. ${ }^{1}$ In addition to this categorization, Modified Pulmonary Index Score (MPIS) was calculated at the time of admission. In the MPIS, 6 categories are evaluated: oxygen saturation, accessory muscle use, inspiratory to expiratory flow ratio, a degree of wheezing, heart rate, and respiratory rate. To each of these 6 measurements or observations was given a score from 0 to 3 (Table 1). ${ }^{11}$

\section{Analysis of blood sample}

Albumin cobalt binding test was used for IMA measurement defined by Bar-Or et al. ${ }^{2}$ The test principle is based on colorimetric determination of the complex of dithiothreitol (DTT) with unbound cobalt. $50 \mu 10.1 \%$ cobalt chloride was added to $200 \mu \mathrm{l}$ patient serum and vortexed and was incubated for 10 minutes to enable albumincobalt binding; $50 \mu 11.5 \mathrm{mg} / \mathrm{mL}$ DTT solution was added to enable reaction of unbound cobalt for 2 minutes. After 2 minutes, a reaction was terminated by adding $0.9 \% \mathrm{NaCl}$. The same method was followed for sample blank, which used distilled water instead of DTT. At the end of the reaction, absorbance levels were determined at $470 \mathrm{~nm}$ in Shimadzu UV-1201 V spectrophotometry. The difference of sample and sample blank was recorded as IMA value. Intraassay and inter-assay CV\% levels of the method were 3.20 and 3.91 respectively.

C-reactive protein levels were measured by immunonephelometry on the BN ProSpec system (Dade Behring Inc.; Illinois, USA). 


\section{Skin prick test}

Patients' skin prick tests were performed when they first applied to our allergy clinic. Skin prick tests with common aeroallergens (Dermatophagoides pteronyssinus, Dermatophagoides farinea, Alternaria alternaria, cockroaches (Blatella germanica), cat dander and dog dander, mixture of grass pollens (Lollium perenne, Dactylis glomerata, Phleum pratense, Anthoxanthum odaratum, Poa pratensis, Festuca eliator, Agrostis vulgaris, Holcus lanatus, Cynodon dactylon, Avena sativa, Avena fatua, Lotus Corniculatus), a mixture of grain pollens (oats, wheat, barley, corn), a mixture of tree pollens (Acer pseudoplanatus, Aesculus hippocastanum, Robinia pseudoacacia, Tilia platyphyllos, Platanus vulgaris), weed-mix pollens (Medicago sativa, Trifolium pratense, Brassica nigra, Urtica dioica, Rumex acetosa), (Stallergenes SA, 92160 Antony, France) were performed using lancet. Skin prick tests were applied on the anterior surface of the forearm. Histamine $(10 \mathrm{mg} / \mathrm{ml})$ and physiological saline were used as positive and negative references, respectively. Skin reactions were evaluated 20 minutes after the application of the skin test, and indurations of $\geq 3 \mathrm{~mm}$ were considered as indicative of a positive reaction.

\section{Statistical analysis}

Sample size was calculated as N: 72. SPSS (Statistical Package for Social Sciences) software package version 15.0 was used for the statistical analysis. Levels for continuous variables were given as either mean \pm standard deviation or as median (interquartile range), based on the normality of distribution. Student $t$ test was used for the comparison of a normal and homogeneous distribution of the parametric levels. Chisquare and Mann Whitney U test were used to compare nonparametric levels. Pearson and Spearman correlation tests were used according to normalities of levels. Wilcoxon Signed Ranks test was used to compare the dependent variables (exacerbations and stable stage). Results were evaluated by accepting the $95 \%$ confidence interval and $\mathrm{p}<0.05$ relevance level.

\section{RESULTS}

A total of 52 children were enrolled in this study. The study group consisted of 26 children with asthma (mean age: $6.05 \pm 2.15$ years; 17 boys and 9 girls) and 26 children as a control group (mean age: $6 \pm 2.12$ years; 15 boys and 11 girls). There were no statistically significant differences in regards to age and gender between patients and control group (respectively, $\mathrm{p}=0.776, \mathrm{p}=0.938$ ). Mean IMA level was $0.45 \pm 0.12$ absorbance units (ABSU) (0.206-0.721) in the study group and $0.32 \pm 0.08$ ABSU (0.145-0.435) in the control group. The difference was statistically significant $(\mathrm{p}=0.001)$.

Nineteen children were receiving antiinflammatory treatment (inhaled steroid and/or montelukast) regularly. The difference was not statistically significant $(p=0.692)$ in comparison of IMA levels in children who did and did not receive anti-inflammatory treatment, although mean IMA level of children who received antiinflammatory treatment was lower than others. Nineteen patients had URTI symptoms before asthma exacerbations. When we compare IMA levels in children who had and had not URTI symptoms, we found that IMA levels were higher, but not statistically significant, in children with URTI symptoms $(p=0.651)$. CRP levels of patients in asthma exacerbation were normal $(<0.5 \mathrm{mg} / \mathrm{dL})$. There was no difference between IMA levels in children who had or had not high

TABle 1. Modified Pulmonary Index Score ${ }^{11}$

\begin{tabular}{|c|c|c|c|c|c|c|c|c|}
\hline & \multicolumn{2}{|c|}{ Heart rate/min } & \multicolumn{2}{|c|}{ Respiratory rate/min } & \multirow{2}{*}{$\begin{array}{l}\text { Accessory } \\
\text { muscle use }\end{array}$} & \multirow{2}{*}{$\begin{array}{l}\text { Inhalation- } \\
\text { exhalation } \\
\text { ratio }\end{array}$} & \multirow[t]{2}{*}{ Wheezing } & \multirow{2}{*}{$\begin{array}{c}\text { Oxygen } \\
\text { saturation } \\
\% \text { (room air) }\end{array}$} \\
\hline & $<3$ years old & $\geq 3$ years old & $<6$ years old & $\geq 6$ years old & & & & \\
\hline 0 & $<120$ & $<100$ & $\leq 30$ & $\leq 20$ & None & $2: 1$ & None & $\geq 96$ \\
\hline 1 & $120-140$ & $100-120$ & $31-45$ & $21-35$ & Mild & $1: 1$ & End expiratory & $93-95$ \\
\hline 2 & $141-160$ & $121-140$ & $46-60$ & $36-50$ & Moderate & $1: 2$ & $\begin{array}{l}\text { Inspiratory and } \\
\text { expiratory wheeze, } \\
\text { good aeration }\end{array}$ & $90-92$ \\
\hline 3 & $>160$ & $>140$ & $>60$ & $>50$ & Severe & $1: 3$ & $\begin{array}{l}\text { Inspiratory and } \\
\text { expiratory wheeze, } \\
\text { decreased aeration }\end{array}$ & $<90$ \\
\hline
\end{tabular}


CRP level ( $p=0.742)$. Twenty-one children had positive skin prick test. Comparison of IMA levels in asthmatic children with and without sensitivity of skin prick test showed no significant difference. $(p=0.111)$. Twenty-three children had mild-moderate severity of asthma, in terms of GINA classification. Comparison of IMA levels, according to severity of asthma exacerbation showed that significantly higher IMA levels were observed in patients with severe asthma exacerbations $(\mathrm{p}=0.009)$ (Table 2$)$.

Mean IMA level of asthmatic children in the asymptomatic period, at least 4 weeks after exacerbations, was $0.41 \pm 0.14$ ABSU (0.1510.699). Mean IMA level in the stable period was significantly higher in the patient group, compared to controls. $(p=0.005)$. IMA levels in asthmatic patients in stable period were lower than patients in exacerbation but this difference was not statically significant $(\mathrm{p}=0.077)$.

Mean MPIS of patients was $7 \pm 2.68$ (3-15). A borderline positive correlation was observed between MPIS and IMA levels $(r=0.376, p=0.05)$.

There was no correlation between IMA levels and admission to the emergency room within last one year, admission to hospital or CRP levels. We observed a negative but not statistically significant relationship between oxygen saturation and IMA level $(p=0.063)$. A positive correlation was observed between IMA level and severity of asthma exacerbations $(\mathrm{r}=0.498, \mathrm{p}=0.010)$ (Figure 1).

\section{DISCUSSION}

We found that IMA levels, in children with asthma exacerbation, were higher than IMA levels in controls in our study. Hypoxia may occur during exacerbation according to the severity of exacerbations. Hypoxia is predominantly seen in severe exacerbations and saturation may decrease lower than $90 \% .{ }^{1}$ We expected higher IMA level in asthma exacerbation, because IMA is related to the ischemia. In our study, we found that IMA levels were higher in children with exacerbated asthma than in controls, consistent with this hypothesis. In the English literature there is no study that evaluates IMA levels in asthmatic patients. In our country, Turkey, a study conducted by Karagol et al. ${ }^{9}$ evaluated IMA levels in asthmatic children at asthma exacerbation and at 14 days after exacerbations. Consistent with Karagol study, we found that IMA levels in patients with asthma exacerbation were significantly higher than in controls. Although IMA levels in stable asthma were lower than exacerbated asthma, that difference was not statistically significant in our study. IMA levels in asymptomatic asthmatic patients were significantly higher than control. Karagol et al. ${ }^{9}$ showed lower, but not statistically significant IMA levels in asymptomatic asthmatic patients compared to control $(p=0.076)$. This discrepancy with our study may be due to differences in study designs. IMA levels were re-measured at least 4 weeks after the asthma exacerbations in our

TABLE 2. Comparison of relation between clinic/laboratory parameters and ischemia modified albumin in asthma exacerbations

\begin{tabular}{|c|c|c|c|}
\hline Parameters & $\mathbf{n}$ & Ischemia modified albumin* & $P$ \\
\hline \multicolumn{4}{|c|}{ Previous treatment intake before the exacerbations, n (\%) } \\
\hline Yes & $19(73.1)$ & $0.44(0.39-0.47)$ & \\
\hline No & $7(26.9)$ & $0.45(0.39-0.58)$ & $0.692^{* *}$ \\
\hline \multicolumn{4}{|c|}{ URTI symptoms before the exacerbations, $\mathrm{n}(\%)$} \\
\hline Yes & $19(73.1)$ & $0.45(0.39-0.55)$ & \\
\hline No & $7(26.9)$ & $0.44(0.37-0.47)$ & $0.651^{* *}$ \\
\hline \multicolumn{4}{|c|}{ Elevation of CRP, n (\%) } \\
\hline Yes & $12(46.2)$ & $0.45(0.34-0.55)$ & \\
\hline No & $14(53.8)$ & $0.44(0.39-0.49)$ & $0.742^{* *}$ \\
\hline \multicolumn{4}{|c|}{ Sensitivity of skin prick tests, $\mathrm{n}(\%)$} \\
\hline Yes & $21(80.8)$ & $0.45(0.42-0.54)$ & \\
\hline No & $5(19.2)$ & $0.33(0.28-0.56)$ & $0.111^{* *}$ \\
\hline \multicolumn{4}{|c|}{ Severity of asthma exacerbations (GINA), n (\%) } \\
\hline Mild-Moderate & $22(84.6)$ & $0.44(0.39-0.46)$ & \\
\hline Severe & $4(15.4)$ & $0.57(0.49-0.69)$ & $0.009^{* *}$ \\
\hline
\end{tabular}

URTI: Upper respiratory tract infection; CRP: C-reactive protein; GINA: Global Initative for Asthma.

*Median (interquartile range); ${ }^{* *}$ Mann Whitney U. 
study, whereas Karagol et al. ${ }^{9}$ re-measured IMA levels at 14 days after the asthma exacerbation. Although Karagol et al. ${ }^{9}$ did not mention the treatment given during exacerbation, IMA levels may be significantly decreased with the treatment (such as systemic steroids) in exacerbation period. Moreover, only patients with mild to moderate exacerbations are included in the study of Karagol et al., ${ }^{9}$ there were several patients with a severe exacerbation in our study.

Although IMA levels initially were thought to be specific for ischemia, some factors such as acidosis, superoxide-radical injury, energydependent membrane disruption and exposure to free iron and copper have been shown to cause IMA formation. ${ }^{4}$ Depending on these factors, in addition to ischemia-related diseases, IMA levels have been found to be increased in inflammatory diseases. ${ }^{5-8}$ Apart from the exacerbation in asthma, airway inflammation continues even during stable period in asthmatic patients. ${ }^{1}$ Increased IMA levels, which are shown to be elevated in inflammatory diseases, are also expected to increase in asthmatic patients during the stable period. We observed that IMA levels in stable asthma higher than controls, consistent with this expectation. Can et al. ${ }^{6}$ evaluated IMA levels in patients with COPD, which is another type of chronic inflammatory disease of airways. Fiftyone patients with COPD and 45 healthy controls were included in that study. They found that serum IMA levels were higher than the control group. These results suggest that IMA may be used to assess inflammation in addition to evaluating hypoxia in airway inflammatory diseases such as asthma and COPD. Our study showed that IMA levels of asthmatic children who received anti-inflammatory treatment were lower, but not statistically significant than children who did not receive anti-inflammatory treatment which supports our hypothesis. Further studies will allow us to make better decisions on this issue.

We used GINA and MPIS exacerbation classifications to evaluate the severity of exacerbations. We observed a borderline positive correlation between MPIS and IMA. Similarly, we observed a positive correlation between



The severity of asthma exacerbations 
GINA and IMA, although we had limited number of patients with severe exacerbation. That result might be related to increased hypoxia and inflammation due to the severity of exacerbation. Unfortunately, we could not evaluate inflammation status. A negative but not statistically significant relationship between oxygen saturation and IMA was observed (r: -0.370, p: 0.063). There was no correlation between severity of exacerbation and IMA levels in the study of Karagol et al. ${ }^{9}$ Similarly there was no correlation between oxygen saturation and IMA levels in our study. However, Karagol et al. ${ }^{9}$ evaluated children with mild and moderate asthma exacerbation only. They did not evaluate children with severe exacerbation. In our study, children with mild and moderate exacerbations were categorized as one group.

There are several limitations of our study. Firstly, we had limited number of patients, because our study is a single-centered and cross-sectional study with limited facilities. The calculated sample size was not reached due to financial difficulties.

Furthermore, we could not evaluate the pulmonary function tests of the patients. Thirdly, we could not evaluate the airway inflammation status with exhaled nitric oxide (NO) measurement, evaluation of sputum cytology, etc. Despite all these limitations, we believe that our study is important because it includes valuable data dispelling the deficiency of the literature.

\section{CONCLUSION}

We found that IMA levels in children with asthma exacerbation were higher than controls. Moreover, IMA levels were higher in stable asthma compared to controls. Positive correlations were shown with IMA levels and the severity of asthma exacerbation. IMA may be helpful to evaluate both inflammation and severity of exacerbation in asthmatic children. However, there is a need for more comprehensive studies on this issue.

\section{REFERENCES}

1. Global Initiative for Asthma: Global Strategy for Asthma Management and Prevention. Updated aug 2015. [Accessed November 17, 2016]. Available at: http:/ / ginasthma.org/ wp-content/ uploads / 2016/01/GINA_Report_2015_ Aug11-1.pdf

2. Bar-Or D, Lau E, Winkler JV. A novel assay for cobaltalbumin binding and its potential as a marker for myocardial ischemia-a preliminary report. J Emerg Med. 2000;19(4):311-5.

3. Toker A, Aribas A, Yerlikaya FH, et al. Serum and saliva levels of ischemia-modified albumin in patients with acute myocardial infarction. J Clin Lab Anal. 2013;27(2):99-104.

4. Sbarouni E, Georgiadou P, Voudris V. Ischemia modified albumin changes - review and clinical implications. Clin Chem Lab Med. 2011;49(2):177-84.

5. Toker A, Karatas Z, Altın H, et al. Evaluation of serum ischemia modified albumin levels in acute rheumatic fever before and after therapy. Indian J Pediatr. 2014;81(2):120-5.

6. Can U, Yerlikaya FH, Yosunkaya S. Role of oxidative stress and serum lipid levels in stable chronic obstructive pulmonary disease. J Chin Med Assoc. 2015;78(12):702-8.

7. Kaplan M, Yuksel M, Ates I, et al. Is ischemia modified albumin a disease activity marker for inflammatory bowel diseases? J Gastroenterol Hepatol. 2016;31(6):1120-5.

8. Ozyazgan S, Andican G, Erman H, et al. Relation of protein oxidation parameters and disease activity in patients with Behçet's disease. Clin Lab. 2013;59(7-8):819-25.

9. Karagol C, Ertoy Karagol HI, Kiyici A, et al. Ischemia Modified Albumin Levels In Children With Acute Asthma Attack. Turkish J Pediatr Dis. 2012;6(3):161-6.

10. Mehmetoglu I, Kurban S, Yerlikaya FH, et al. Obesity is an independent determinant of ischemia-modified albumin. Obes Facts. 2012;5(5):700-9.

11. Carroll CL, Sekaran AK, Lerer TJ, et al. A modified pulmonary index score with predictive value for pediatric asthma exacerbations. Ann Allergy Asthma Immunol. 2005;94(3):355-9. 